\title{
Study of Metal-Carbon Nanofiber Interfaces for On-chip Interconnect Applications
}

\author{
Y. Ominami ${ }^{1}$, Q. Ngo ${ }^{1,2}$, H. Yoong ${ }^{1}$, A.J. Austin ${ }^{1}$, A.M. Cassell ${ }^{2}$, B.A. Cruden ${ }^{2}$, J. Li $^{2}$, \\ M. Meyyappan ${ }^{2}$, and C.Y. Yang ${ }^{1}$ \\ ${ }^{1}$ Center for Nanostructures, Santa Clara Univ., 500 El Camino Santa Clara CA, USA \\ ${ }^{2}$ NASA Ames Research Center, Center for Nanotechnology, Moffett Field, CA, USA
}

Nanoelectronic devices fabricated using carbon nanotubes (CNT) and nanofibers (CNF) are prospects for both transistor and interconnect applications because of their unique electronic properties [1-3]. For interconnect applications, it is important that the interface resistance between $\mathrm{CNF}$ and metal is minimized. Therefore the development of a low-resistance interface requires critical structural and chemical information from high-resolution microscopy. In this work, we grow vertically aligned CNFs on various metal layers. The interfacial structures are characterized using a scanning transmission electron microscope (STEM) equipped with energy-dispersive x-ray spectroscopy (EDX) capability.

The CNFs are vertically grown on the metal layer by plasma-enhanced chemical vapor deposition (PECVD) as described in [4]. Scanning electron microscopy (SEM) reveals vertically aligned CNF arrays as shown in Figure 1(a). Cr, Pt, or Ti is deposited as the underlayer metal, which has been shown to yield low CNF-metal contact resistance [5], and Ni or Pd is used as the catalyst material. Subsequently, $\mathrm{SiO}_{2}$ is deposited using tetraethoxysilane (TEOS) CVD and the sample is treated with chemical mechanical polishing (CMP) [3]. These steps are taken to protect the CNF from damage during STEM sample preparation. The resulting structure is shown in Figure 1(b). From current-sensing atomic force microscopy (CSAFM) and wafer-probe electrical measurements, the resistance of Ni-catalyzed CNFs is found to be higher than that of Pd-catalyzed CNFs.

In order to obtain CNF interface images using STEM, it is important to maintain the integrity of the forest-like arrays of CNFs after sample preparation. Therefore micro-sampling technique and sputtering using a $10 \mathrm{kV}$ focused ion beam are used to prepare the STEM sample. The STEM images of each sample are shown Figure 2. The high-resolution STEM image of a Ni-catalyzed CNF is shown in Figure 3(a). The interface between CNF and metal consists of many graphene layers that are almost parallel to the silicon substrate surface. The spacing between adjacent graphene layers measured with high-resolution STEM is $0.34 \mathrm{~nm}$, which is consistent with that of turbostratic stacking of graphene layers in nanofibers [6]. This interfacial region has a thickness approaching $100 \mathrm{~nm}$, giving rise to significant contact resistance. In contrast, the interfacial structure of Pdcatalyzed CNFs shows multiwall CNT-like morphology with each wall nearly perpendicular to the substrate, as indicated in Figure 3(b). Thus, as electrons flow in the basal plane of each graphene layer, parallel to the nanofiber axis, the resulting CNF-metal contact resistance of the Pd-catalyzed sample is substantially lower than that of the Ni-catalyzed one as our measured resistances indicate.

[References]

[1] F. Kreupl et al., Microelectrom. Eng. 64, 399 (2002)

[2] R.Martel et al., Appl. Phys. Lett. 73, 2447 (1998)

[3] J.Li et al., Appl. Phys. Lett. 82, 2491 (2003)

[4] B.A.Cruden et al., J. Appl. Phys. 94, 4070 (2003)

[5] Q. Ngo et al., IEEE Transactions on Nanotechnology, 3, No.2, 311 (2004)

[6] H. Cui et al., Appl. Phys. Lett. 84, 4077 (2004) 

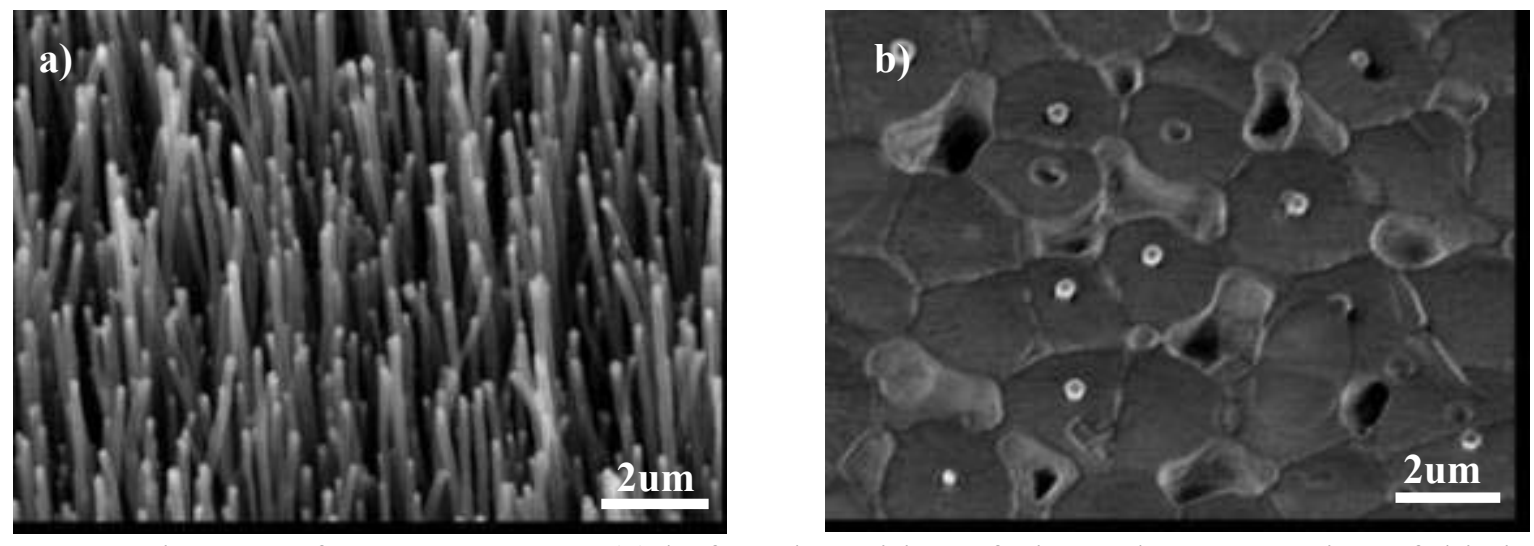

Fig.1 SEM images of as-grown CNFs (a) before deposition of $\mathrm{SiO}_{2}$. The perspective of this image is $45^{\circ}$. (b) After deposition of $\mathrm{SiO}_{2}$ and CMP. The perspective of this image is $25^{\circ}$. The lighter areas represent the CNF tips.
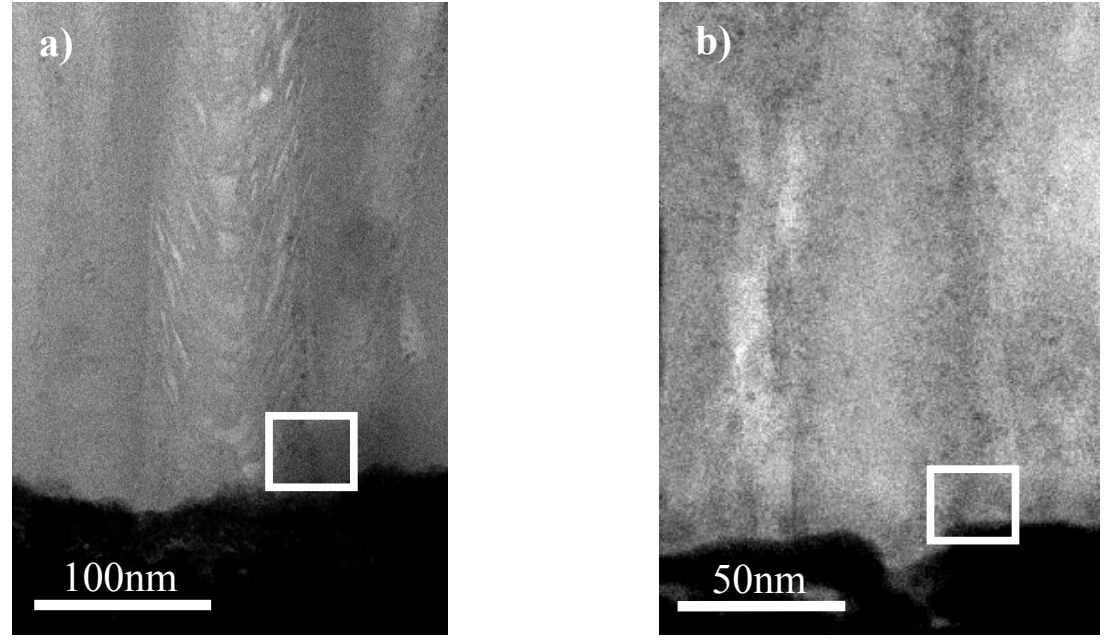

Fig.2 STEM images of (a) a Ni-catalyzed CNF (b) a Pd-catalyzed CNF. The solid lines indicate the observation area.
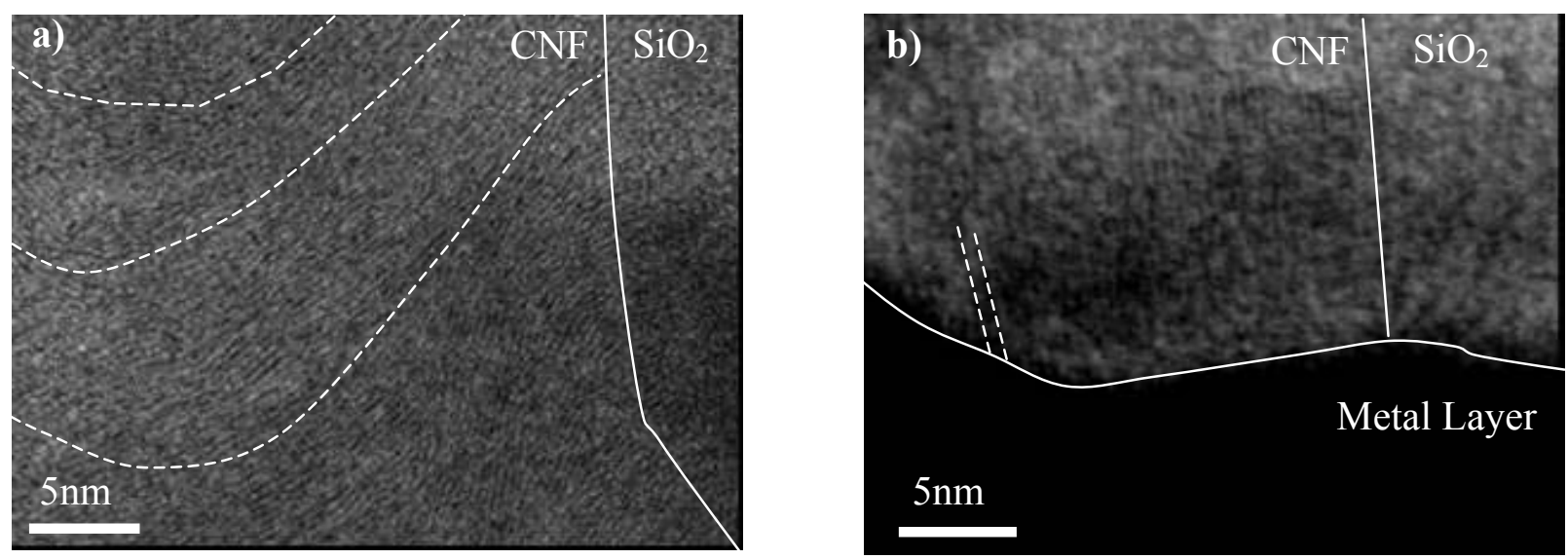

Fig.3 High resolution-STEM images of (a) the bottom of Ni-catalyzed CNF (b) the interface of Pd-catalyzed CNF. The solid lines indicate the boundaries among $\mathrm{CNF}, \mathrm{SiO}_{2}$, and metal layer. The broken lines indicate some graphene layers' orientations. 